\title{
Optimal Pricing Strategy for Multichannel Healthcare Services
}

\author{
Wei Wang \\ School of Management \\ University of Science and Technology of China \\ ww951010@mail.ustc.edu.cn \\ Hanyu Xiao \\ School of Management \\ University of Science and Technology of China \\ xhyzkj@mail.ustc.edu.cn
}

\author{
Xiao Shi \\ School of Management \\ University of Science and Technology of China \\ shixiao@mail.ustc.edu.cn \\ Xiabing Zheng \\ School of Management \\ University of Science and Technology of China \\ isxzheng@ustc.edu.cn
}

\begin{abstract}
As a combination of online and offline channel services, multichannel healthcare services currently play important roles in helping consumers solve their health problems. In this study, we establish a stylized model to investigate how healthcare service providers should price in multi-channels and when consumers should choose online service, taking misdiagnosis rate and the severity of disease problems into account. Our results show that the prices of the online channel and offline channel can increase when the misdiagnosis rate is low and minor problem inspection rate online is high. Moreover, when the diagnosis rate is high, the profit of online channel would increase, and then improve the profit of multichannel service. These findings provide insights for the theoretical research of online healthcare service and practice management on pricing strategies in multichannel healthcare services.
\end{abstract}

\section{Introduction}

Statistics showed that the number of medical practitioners in China has steadily increased from 2.08 million in 2008 to 3.39 million in 2017 [17, 26]. In addition, the number of total treatments has raised from 4.9 billion in 2008 to 8.1 billion in 2017 [9]. These data imply that the traditional offline healthcare system is over demand and under tremendous pressure. Therefore, an imbalance between the large amounts of consumers and the insufficient amounts of physicians exists. With the development of information technology, online healthcare service occurs [27]. As a supplement to traditional offline hospitals, online healthcare channel is gradually adopted by consumers [37].
Multichannel healthcare services involve both online and offline healthcare service to provide an additional amount of services than the traditional offline one [24]. In the traditional offline healthcare service, consumers first need to queue up for an appointment at the hospital. Then, physicians have a detailed and comprehensive knowledge of the consumers' physical condition after the face-to-face inquiring and required physical testing. Finally, physicians provide consumers further diagnostic solutions or other suggestions. Therefore, consumers need to spend a substantial amount of time and energy to receive the offline channel healthcare service. In addition, the supply quantity of traditional offline healthcare service is limited by the number of physicians, because the limited provision of physicians can only serve a limited number of consumers in one day.

In the online healthcare service, consumers need not have to wait in line as generally practiced in offline hospital. However, owing to the privacy concern of sharing health information online, consumers may be reluctant to tell their actual physical state to protect their privacy [19]. Under this information asymmetry circumstance, physicians may not know exactly about the consumer's actual physical condition, which will lead to misdiagnosis and ineffective problem solving $[22,25]$. Moreover, in some cases of healthcare problems, because of unavailable inspection equipment or technical limitations in the online channel, physicians cannot provide accurate solutions to consumers' health problems online. In these cases, the physicians will advise consumers that additional offline service can offer a further accurate diagnosis.

Some of the healthcare service providers still focus on offering service in traditional offline hospitals, whereas others may expand service to online channels, such as online healthcare communities [14]. Considering the differences between the online and 
offline channels in the healthcare service market, we propose the following research questions:

1. How do service providers set the price of online and offline channel respectively to maximize the profits of the multichannel healthcare service market?

2. When do consumers prefer to choose online channel to seek a healthcare service?

3. How will the profit of online channel affect the profits of offline channel and multichannel?

From game theory $[15,16,33]$ and utility maximization method [20], we establish a stylized model to address the issues of resource allocation and pricing strategies in online and offline channels. In our model, we not only investigate the multichannel services in the healthcare industry, but also take the misdiagnosis rate and the severity of disease problems into consideration. It also provides management advice for the healthcare service market.

\section{Literature review}

\subsection{Multichannel healthcare services}

The recent growth of multichannel service is correlated to the booming development of information technology and e-services [4, 35, 27]. Offline channel and online channel are the two main ways of multichannel services. Previous studies have given different definitions of multichannel. Melis et al. [13] regarded multichannel as a combination of online stores and offline shops, which become an overall retailing mode. Berman and Thelen [2] stated that the integration between online and offline channels is the multichannel services, which is used in retail industry. However, healthcare service is different from retailing production [20]. According to Melis et al. [13], we define multichannel as a combination of online and offline channels, which can coexist in the healthcare service market.

To manage multichannel retail market, prior studies have elucidated that a proper multichannel strategy can increase consumer loyalty [3] and improve profit [29]. In addition, Zhang et al. [11] listed some various key factors of multichannel retail service strategy, such as organizational structure, data integration, consumer analysis, and evaluation to show that how multichannel retailing evolve over time. Concerning consumers' behavior in multichannel market, $\mathrm{Gao}$ and $\mathrm{Su}$ [5] investigated consumers' purchasing behavior in both online and offline channels.

Regarding the healthcare industry, many researchers focused on the either offline healthcare service [23] or online healthcare service [14]. For offline healthcare channel, researchers found that there is a significant spillover effect between regional offline hospitals [6]. Some studies pointed out that the use of information technology will influence the quality of healthcare service [1, 34]. For online healthcare channel, researchers figured out some driving factors in online healthcare consultation $[18,28]$, such as the use of social media and doctor-patient relationship. Guo et al. [28] also found that the quantity of online consultation is related to the comment and degree of expertise as well as online price. Gradually, researchers began to investigate the multichannel healthcare service, which include both online channel and offline channel. Wu and Lu [7] stated that physicians with high reputation can strength the relationship between offline hospitals communication and online channel interaction. Akçura et al. [21] developed a mathematical model to explore the impact of online channel on service market, revealing that high-quality providers cannot offer a sufficiently high-quality online service. Also, Akçura and Ozdemir indicated that management strategy of online and offline service market should be different in multichannel healthcare service [20]. Previous studies mainly consider pricing from the perspective of service providers. Our study explores the impact of consumers' channel choice on online and offline channel's pricing strategies from the perspective of consumers.

\subsection{Misdiagnosis}

Generally, consumers are concerned about the quality of healthcare service to solve health problems effectively. Physicians in traditional offline healthcare service can know comprehensively about consumers' physical condition through face-to-face inquiry and physical examination. While in online healthcare channel, consumers can share limited information to physicians via online interaction, such as the discomfort of body. Therefore, there exist information incomplete and information asymmetry in the online healthcare service. And incomplete information or information asymmetry can lead to physicians' misdiagnosis [8]. Misdiagnosis is the mistakes or inaccuracies of the physician's diagnosis. Misdiagnosis has an adverse effect on the quality of healthcare service and makes consumers' problems unsolved. Hyndman and Ozerturk [12] explored the relationship between consumers' professional knowledge and the probability of being misdiagnosed in the process of healthcare service. They found that if physicians cheat the knowledgeable customers, market efficiency would reduce [12]. Also, some studies have suggested that searching for a second opinion, which means having a re-examination, can significantly reduce misdiagnosis [32].

In online healthcare service, existed studies mainly investigated the quality of online healthcare service, 
such as consumers' loyalty [30] and consumers' satisfaction [36]. Regarding misdiagnosis in online healthcare service, researchers figured out that perceived usefulness of using smart healthcare service may decrease online misdiagnosis [10]. Researchers found that using artificial intelligence-based medical diagnosis support system in complex situations online would improve the accuracy and effectiveness of physicians' diagnosis [31]. However, there is few research on studying the impact of online misdiagnosis in multichannel healthcare services. Our study takes the physician's online misdiagnosis into account to develop the following model.

\section{Model}

Healthcare service market consists of consumers, who face problems, and physicians, who provide solutions or suggestions to consumers' problems. The current study assumes that all consumers know they have some health problems but are unaware of the exact nature of their problems. Thus, consumers should attempt to seek solutions and solve their problems from either offline or online channels.

The offline channel. Consumers who seek for offline channel service must visit hospitals. The distance cost of receiving offline services is $\varepsilon$. When $\varepsilon$ is large, the consumers will perceive the distance cost as excessively high to go offline. Then physicians communicate with consumers through face-to-face inquiry and examine the consumers' physical condition so that they can offer solutions to consumers' health problems. The marginal cost of serving a consumer in the offline hospital is $c$. In light of Akçura and Ozdemir [20], we assume that $\varepsilon$ and $c$ are both distributed uniformly across $[0,1]$.

If the price of offline is $p_{f}$, then, the utility of consumer who seeks and purchases service from the offline physician is given by:

$$
U_{f}=V-p_{f}-\varepsilon \text {, }
$$

where $\mathrm{V}$ is the base valuation. We assume that $\mathrm{V}$ is sufficiently high to allow at least some consumers to look for service in equilibrium.

The online channel. Face-to-face interactions (i.e., offline channel) in a private physician's office require consumers to disclose all relevant information and undergo physical examination for a thorough knowledge of their health problems. However, in online channel, physicians will receive less information with no inspection. In addition, Akçura and Srinivasan [19] have shown that consumers with privacy concern can only share limited information with others online. Such condition may decrease the accuracy of physicians' diagnosis in the online channel. Thus, we conjecture that misdiagnosis will result in problems being unsolved in online channel than in offline healthcare service.

When the physician misdiagnoses, the consumer will receive an ineffective solution. We assume that consumers will receive an effective diagnosis, so $h$ denotes the effective diagnosis rate in the online channel, where $0<h<1$. Accordingly, consumers would receive a probability $1-h$ of misdiagnosis. We conjecture that the misdiagnosis will result in problems unsolved. After being misdiagnosed in online channel, consumers would not believe online channel at all and consider if they should seek subsequent offline service or leave the market and relinquish any treatment. Because of the misdiagnosis, the consumers' utility of the online channel will be reduced. Therefore, $K$ denotes the utility loss after misdiagnosis.

If the physician diagnoses effectively in the online channel, the physician will inform the consumer the nature of the problem and offer the consumer some suggestions. The diagnosis result will help consumers estimate the severity degree, whether major or minor. Major problems refer to more complex and severe diseases requiring further examination to identify their exact nature, such as chronic disease, cancer, cardiovascular disease, and cerebrovascular diseases. Minor problems are classified as sicknesses that are obvious and easy to judge and solve, such as influenza, cough, and diarrhea. Physicians can give further reasonable treatments to minor problems. However, to successfully solve consumers' major problems, physicians need consumers' medical history, inspection results, and several other related information, which only the offline environment can provide. To incorporate the severity degree of health problem in the online channel, we assume that a consumer has a minor problem online with a probability $\alpha$, where $0<\alpha<1$. When consumers first select the online channel for healthcare service and obtain the diagnosis result of a minor problem, the physician will solve the consumer's problem. When consumers select the online channel for healthcare service first and obtain the diagnosis result of a major problem, they will consider the following behavioral decision (i.e., visit the offline hospital to test and solve problems or abandon any offline treatment). Regardless of the type of healthcare problems is major or minor, whether major or minor, we regard these problems would be solved via an offline channel.

If the physician charges the price of the online channel $p_{o}$, then, the utility of consumer who purchases the service from the online physician is given by:

$$
\begin{gathered}
U_{o}=(1-h)\left(\mathrm{V}-p_{o}-\mathrm{K}-p_{f}-\varepsilon\right)+h \alpha\left(\mathrm{V}-p_{o}\right) \\
+h(1-\alpha)\left(\mathrm{V}-p_{o}-p_{f}-\varepsilon\right),
\end{gathered}
$$

where the first, second, and third terms are for the utility of receiving misdiagnosis service, the utility of obtaining the effective service and the diagnosis of a 
problem as a minor problem, and the utility of obtaining the effective service and the diagnosis of a problem as a major problem, respectively.

Simplifying the formula above, we will obtain:

$$
\begin{aligned}
U_{o}= & (1-h \alpha)\left(V-p_{o}-p_{f}-\varepsilon\right)+h \alpha V \\
& -p_{o}-(1-h) K .
\end{aligned}
$$

Table 1 details the notation used in this study.

Table 1. Notations and definitions

\begin{tabular}{|c|l|}
\hline Notation & \multicolumn{1}{c|}{ Definition } \\
\hline $\mathrm{V}$ & The base valuation \\
\hline$p_{f}$ & Service price of offline channel \\
\hline$p_{o}$ & Service price of online channel \\
\hline$c$ & $\begin{array}{l}\text { The marginal cost of serving a } \\
\text { consumer in offline channel }\end{array}$ \\
\hline$\varepsilon$ & Distance cost of getting offline service \\
\hline $\mathrm{K}$ & Utility loss after misdiagnosis \\
\hline$h$ & $\begin{array}{l}\text { Effective diagnosis rate in online } \\
\text { channel }\end{array}$ \\
\hline$\alpha$ & $\begin{array}{l}\text { Minor problem inspection rate in online } \\
\text { diagnosis }\end{array}$ \\
\hline$U_{f}$ & The utility of consumers offline \\
\hline$U_{0}$ & The utility of consumers online \\
\hline
\end{tabular}

Consumers in the healthcare service market have the following four options: (1) directly seeking offline service and solving the problem; (2) choosing online service first; obtaining offline face-to-face service or leaving the market once misdiagnosed; (3) if diagnosed with minor problems, it would be solved via an online channel; or (4) if diagnosed with major problems, it would be solved in an offline channel or leave the market. Figure 1 shows the process and decisions for the entire service in the market.

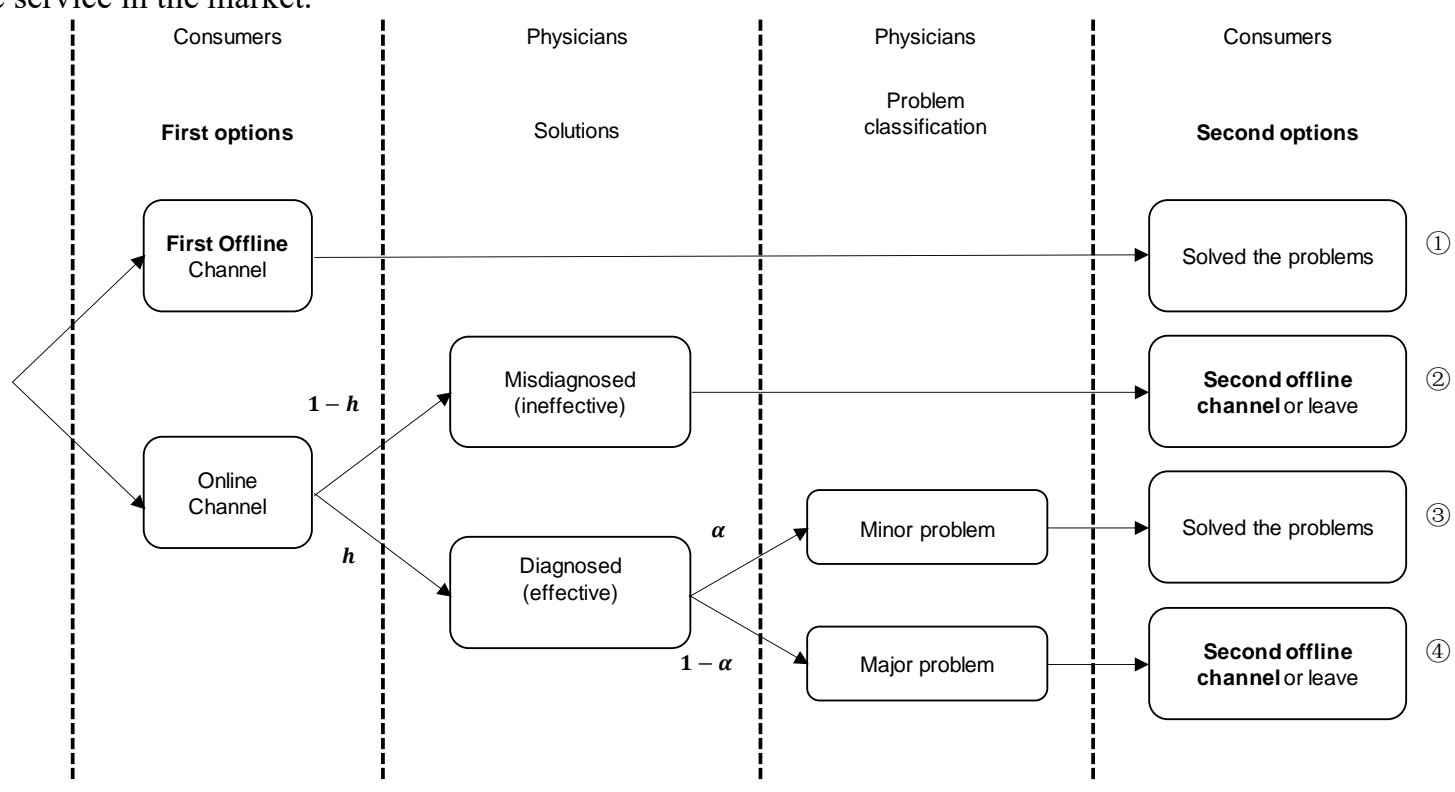

Figure 1. The process and decisions for the entire service in the market

\section{Analysis}

First, the consumer considers whether the initial visit is offline or online. The consumer who is indifferent between offline and online channels is located at $\varepsilon_{0}$. Solving Equations (3.1) and (3.3) for $\varepsilon_{0}$, we have:

$$
\varepsilon_{0}=\frac{p_{o}+(1-h) K}{h \alpha}-p_{f} \text {. }
$$

Second, if the consumer lives far away from the offline hospital, he would prefer online service because of the high transportation cost. However, when facing an ineffective service or a major problem, the consumer must consider the utility of the offline channel. The consumer, who lives farther away:

$$
\varepsilon_{1}=V-p_{f}
$$

will find it too costly to seek an offline service, so he will leave the market without any solutions. The consumer who lives closer than $\varepsilon_{1}$ will prefer offline service to solve their problems.

Thus, the profit function for the service provider is:

$$
\begin{aligned}
\Pi= & \varepsilon_{0}\left(p_{f}-c\right)+\left(1-\varepsilon_{0}\right) p_{0} \\
& +(1-h \alpha)\left(\varepsilon_{1}-\varepsilon_{0}\right)\left(p_{f}-c\right),
\end{aligned}
$$

where the first, second, and third terms are for the profits from offline (i.e., first) service, online service, and offline (i.e., second) service, respectively.

Further simplifying the above equations, we thus have:

$$
\begin{aligned}
\Pi= & \varepsilon_{0}\left(p_{f}-c\right)+\left(1-\varepsilon_{0}\right) p_{0} \\
& +(1-h \alpha)\left(\varepsilon_{1}-\varepsilon_{0}\right)\left(p_{f}-c\right) .
\end{aligned}
$$

Derivatives of $p_{o}$ and $p_{f}$ are obtained respectively from Formula (3.7). We can obtain the following optimal equilibrium prices of the online channel and offline channel: 


$$
\begin{aligned}
& p_{o}^{*}=\frac{h \alpha V-(1-h) K}{2}+\frac{h \alpha}{2(1-h \alpha)} . \\
& p_{f}^{*}=\frac{V+c}{2}+\frac{h \alpha}{2(1-h \alpha)} .
\end{aligned}
$$

Proposition 1. $p_{o}^{*}$ and $p_{f}^{*}$ increase with the increase in $\alpha$ and $h$, respectively.

When $\alpha$ or $h$ are higher, both the online and offline prices are higher. When some minor problems become prevalent, more consumers can obtain a solution online; then, the online channel will gain additional trust from consumers. Therefore, service providers can raise the price of the online channel to make extra money. When physicians attain an accurate diagnosis, customers will trust the online channel and purchase healthcare service more from online; thus, the price of the online channel increases.

By substituting $p_{o}^{*}$ and $p_{f}^{*}$ for Equations (3.4) and (3.5), we can obtain $\varepsilon_{0}$ and $\varepsilon_{1}$. Furthermore, we will obtain the demand of offline market and online market and ultimately the unresolved demand.

Online demand:

$$
\begin{aligned}
& h \alpha\left(1-\varepsilon_{0}\right)=\frac{h \alpha(1+c)-(1-h) K}{2} . \\
& \text { Totally offline demand: } \\
& \varepsilon_{1}-h \alpha\left(\varepsilon_{1}-\varepsilon_{0}\right)=\frac{(1-h \alpha) V+(1-h) K-c}{2} .
\end{aligned}
$$

Unsolved demand:

$$
(1-h \alpha)\left(1-\varepsilon_{0}\right)=1-\frac{h \alpha+(1-h \alpha)(v-c)}{2} \text {. }
$$

Proposition 2. When $\frac{\mathrm{K}}{\mathrm{K}+\alpha(1+\mathrm{c})}<\mathrm{h}<1$, consumers will choose the online channel.

In this case, service providers should raise the offline price to obtain sufficient profit. Thus, misdiagnosis should not be too high, otherwise, consumers will not choose an online channel.

According to the optimal pricing, we can also have the profit via online and offline channels.

Overall profit:

$$
\begin{aligned}
\Pi^{*}= & \frac{1}{4}\left[(v-c)^{2}(h \alpha V-K+h K)(2-V+2 c)-\right. \\
& \left.(v-c)^{2}(h \alpha V-K+h K) \frac{K-h K}{h \alpha}+\frac{h \alpha}{1-h \alpha}\right] .
\end{aligned}
$$

Online profit:

$$
\begin{aligned}
& \Pi_{o}^{*}=\left(1-\varepsilon_{0}\right) p_{0} \\
&=\left(\frac{1+c}{2}-\frac{(1-h) K}{h \alpha}\right)\left[\frac{h \alpha V-(1-h) K}{2}+\frac{h \alpha}{2(1-h \alpha)}\right] . \\
& \text { Offline profit: } \\
& \Pi_{f}^{*}=\varepsilon_{0}\left(p_{f}-c\right)+(1-h \alpha)\left(\varepsilon_{1}-\varepsilon_{0}\right)\left(p_{f}-c\right) \\
&=\left(\frac{1-c}{2}+\frac{(1-h) K}{h \alpha}\right)\left[\frac{V-c}{2}+\frac{h \alpha}{2(1-h \alpha)}\right]+ \\
& \frac{(V-c)(1-h \alpha)}{2}\left[\frac{V-1}{2}-\frac{h \alpha}{2(1-h \alpha)}-\frac{(1-h) K}{h \alpha}\right]+ \\
& \frac{h \alpha}{2}\left[\frac{V-1}{2}-\frac{h \alpha}{2(1-h \alpha)}-\frac{(1-h) K}{h \alpha}\right] .
\end{aligned}
$$

Proposition 3. As $h$ increases, online profits increase, offline profits decrease, and overall profits increase.

As the accuracy of the diagnosis increases, the online channel gains additional customers, and fewer customers will choose the offline channel. Therefore, the profit of the online channel will rise, and the profit of the offline channel will decrease. For the entire market, because the allocation of resources is effective, the profits of the entire market will increase, thereby improving social welfare.

\section{Discussion}

\subsection{Interpretation of findings}

In this study, we investigate the impact of misdiagnosis rate and the severity of the health problems on the multichannel healthcare service market. Then we obtain the following conclusions.

First, service providers must raise their price in online and offline channels according to effective diagnosis rate and the probability of minor problems. The effective diagnosis rate and the probability of minor problems also positively affect the optimal pricing of both online and offline channels. Second, when the rate of online misdiagnosis under a certain degree, consumers will prefer the online channel. Third, when the misdiagnosis rate is low, the online channel will attract other consumers, which results in the high profit of online channel and low profit of the online channel. However, under this circumstance, the entire multichannel profit increases. Thus, providing online channel service and alleviating offline treatment pressure are beneficial for multichannel service providers.

\subsection{Implications for research}

The results of this study have the potential to make several contributions to research. First, we think that this study covers the shortage of multichannel service in the healthcare industry. Although literature exists about multichannel services in retail industry [5], service industry, such as multichannel healthcare services, remains lacking.

Second, the study of the misdiagnosis rate of the online channel is relatively new to supply chain research. Despite some studies on the relationship between misdiagnosis and customer satisfaction [36], only a few studies have considered the impact of misdiagnosis on pricing strategy in the multichannel market.

Third, this study provides insights into the effect of different kinds of situations on users' channel preferences. We find that minor problem inspection rate has a significant positive effect on customers' choice of the online channel.

\subsection{Implications for practice}


In practice, we put forward some management suggestions from three aspects. First, for the government, we propose that the government should strengthen the control of healthcare industry and pay attention to the quality of online healthcare service to reduce the probability of misdiagnosis so that other customers' problems can find effective solutions through online and offline channels. Second, for the healthcare industry, we suggest that service providers can increase the investment to develop information technology, which can improve the quality of online healthcare service and reduce the pressure of excessive demand for offline healthcare service. Through the effective and rational allocation of resources, additional profits can be obtained. Third, for consumers, when the minor problems are prevalent, online service can help cut down on expenditure, save time, and improve service efficiency. We suggest that consumers who can solve minor problems at a lower cost should access the online channel instead of the offline channel, whereas consumers who must solve major problems at a higher cost can directly access the offline channel.

\subsection{Limitations and future research}

Overall, this study settles the question of how to manage multi-channel to allocate healthcare service resources rationally, which is conducive to multichannel operation and sustainable development of resources. However, some limitations are identified in in our study. First, we only consider dividing the disease into size problems and less on detailed problems. Different types of problems have different treatment methods. For example, chronic disease may require repeatedly online inquiries and long-term inspection, which will be a process of multiple decision making. Second, we did not consider the impact of healthcare conditions in different regions on the quality of service. For example, in relatively poor areas, the offline channel may not solve the problem, but online channel may have higher service. Third, we only consider the general situation without differentiating the effect of various cultures. Future research can consider cultural factors.

\section{Acknowledgements}

This work was supported by the National Natural Science Foundation of China (No. 71701195, 71631006) and the Foundation for Innovative Research Groups of National Natural Science of China (Grant No. 71921001).

\section{References}

[1] A. Pinsonneault, S. Addas, C. Qian, V. Dakshinamoorthy, and R. Tamblyn, "Integrated Health Information Technology and the Quality of Patient Care: A Natural Experiment", Journal of Management Information Systems, 34 (2017), pp. 457-486.

[2] B. Berman and S. Thelen, "A Guide to Developing and Managing a Well-Integrated Multi-Channel Retail Strategy", International Journal of Retail \& Distribution Management, 32 (2004), pp. 147-156.

[3] D.W. Wallace, J.L. Giese, and J.L. Johnson, "Customer Retailer Loyalty in the Context of Multiple Channel Strategies", Journal of Retailing, 80 (2004), pp. 249-263.

[4] E. Pantano and M. Viassone, "Engaging consumers on new integrated multichannel retail settings: Challenges for retailers", Journal of Retailing and Consumer Services, 25 (2015), pp. 106-114.

[5] F. Gao and X. Su, "Omnichannel Retail Operations with Buy-Online-and-Pick-up-in-Store”, Management Science, 63 (2016), pp. 2478-2492.

[6] H. Atasoy, P.Y. Chen, and K. Ganju, "The Spillover Effects of Health It Investments on Regional Healthcare Costs", Management Science, 64 (2017), pp. 2515-2534.

[7] H. Wu and N. Lu, "Online Written Consultation, Telephone Consultation and Offline Appointment: An Examination of the Channel Effect in Online Health Communities", International Journal of Medical Informatics, 107 (2017), pp. 107-119.

[8] I. Alger and F. Salanie, "A Theory of Fraud and Overtreatment in Experts Markets", Journal of Economics \& Management Strategy, 15 (2006), pp. 853-881.

[9] iresearch, "Analysis on the Current Situation and Development Trend of China's Big Data Industry of Health Care in 2018", https://www.iresearch.com.cn/Detail/report?id=3218\&isfree= 0, May, 2018.

[10] J. Pan, S. Ding, D. Wu, S. Yang, and J. Yang, "Exploring behavioural intentions toward smart healthcare services among medical practitioners: A technology transfer perspective", International Journal of Production Research, 57(2019), pp. 5801-5820.

[11] J. Zhang, P.W. Farris, J.W. Irvin, T. Kushwaha, T.J. Steenburgh, and B.A. Weitz, "Crafting Integrated Multichannel Retailing Strategies", Journal of Interactive Marketing, 24 (2010), pp. 168-180.

[12] K. Hyndman and S. Ozerturk, "Consumer Information in a Market for Expert Services", Journal of Economic Behavior \& Organization, 80 (2011), pp. 628-640. 
[13] K. Melis, K. Campo, E. Breugelmans, and L. Lamey, "The Impact of the Multi-Channel Retail Mix on Online Store Choice: Does Online Experience Matter?", Journal of Retailing, 91 (2015), pp. 272-288.

[14] L. Chen, A. Baird, and D. Straub, "Fostering Participant Health Knowledge and Attitudes: An Econometric Study of a Chronic Disease-Focused Online Health Community", Journal of Management Information Systems, 36 (2019), pp. 194-229.

[15] L.C. Thomas, "Games, theory and applications", Courier Corporation, 2012.

[16] M. Bhattacharryya and S.S. Sana, "A Mathematical Model on Eco-Friendly Manufacturing System under Probabilistic Demand", RAIRO-Oper. Res., (2019).

[17] Ministry of Health, "Ministry of Health Issued Statistics Bulletin on Health Development in China in 2008", http://www.gov.cn/gzdt/2009-04/29/content_1299547.htm, April 29, 2009.

[18] M.L. Antheunis, K. Tates, and T.E. Nieboer, "Patients' and Health Professionals' Use of Social Media in Health Care: Motives, Barriers and Expectations", Patient Education and Counseling, 92 (2013), pp. 426-431.

[19] M.T. Akçura and K. Srinivasan, "Research Note: Customer Intimacy and Cross-Selling Strategy", Management Science, 51 (2005), pp. 1007-1012.

[20] M.T. Akçura and Z.D. Ozdemir, "A Strategic Analysis of Multi-Channel Expert Services", Journal of Management Information Systems, 34 (2017), pp. 206-231.

[21] M.T. Akçura and Z.D. Ozdemir, and S. Jain, "Expert Competition and the Internet", International Journal of Electronic Commerce, 18 (2013), pp. 11-44.

[22] M. Gaynor, "Issues in the industrial organization of the market for physician services", Journal of Economics \& Management Strategy, 3(1994), pp. 211-255.

[23] N. Liu, S.R. Finkelstein, M.E. Kruk, and D. Rosenthal, "When waiting to see a doctor is less irritating: Understanding patient preferences and choice behavior in appointment scheduling”, Management Science, 64 (2017), pp. 1975-1996.

[24] N. M. Modak and P. Kelle, "Managing a Dual-Channel Supply Chain under Price and Delivery-Time Dependent Stochastic Demand", European Journal of Operational Research, 272 (2019), pp. 147-161.

[25] N. Yaraghi, R.D. Gopal, and R. Ramesh, "Doctors' Orders or Patients' Preferences? Examining the Role of Physicians in Patients' Privacy Decisions on Health Information Exchange Platforms", Journal of the Association for Information Systems, 20 (2019), p. 14.
[26] People's daily, "The Number of Medical and Health Personnel in China is Steadily Increasing--The Number of Medical Practitioners per 1000 Population is 2.4", http://politics.people.com.cn/n1/2018/0816/c100130231326.html, August 16, 2018.

[27] P. Luo, K. Chen, C. Wu, and Y. Li, "Exploring the social influence of multichannel access in an online health community", Journal of the Association for Information Science and Technology, 69(2018), pp. 98-109.

[28] S. Guo, X. Guo, X. Zhang, and D. Vogel, "Doctor-Patient Relationship Strength's Impact in an Online Healthcare Community", Information Technology for Development, 24 (2018), pp. 279-300.

[29] S.A. Neslin, D. Grewal, R. Leghorn, V. Shankar, M.L. Teerling, J.S. Thomas, and P.C. Verhoef, "Challenges and Opportunities in Multichannel Customer Management", Journal of Service Research, 9 (2006), pp. 95-112.

[30] S. Annamdevula and R. S. Bellamkonda, "The Effects of Service Quality on Student Loyalty: The Mediating Role of Student Satisfaction", Journal of Modelling in Management, 11 (2016), pp. 446-462.

[31] W. Fan, J. Liu, S. Zhu, P.M. Pardalos, "Investigating the impacting factors for the healthcare professionals to adopt artificial intelligence-based medical diagnosis support system (AIMDSS)“, Annals of Operations Research, (2018), pp. 1-26.

[32] W. Mimra, A. Rasch, and C. Waibel, "Second opinions in markets for expert services: Experimental evidence", Journal of Economic Behavior \& Organization, 131 (2016), pp. $106-125$

[33] W. Zhou, Q. Wan, and R.Q. Zhang, "Choosing among Hospitals in the Subsidized Health Insurance System of China: A Sequential Game Approach", European Journal of Operational Research, 257 (2017), pp. 568-585.

[34] Y.K. Lin, M. Lin, and H. Chen, "Do Electronic Health Records Affect Quality of Care? Evidence from the Hitech Act”, Information Systems Research, 30 (2019), pp. 306-318.

[35] Y. Lu, A. Gupta, W. Ketter, and E. Heck, "Exploring bidder heterogeneity in multichannel sequential B2B auctions", MIS Quarterly, 40(2016), pp. 645-662.

[36] Y. Politis, A. Giovanis, and S. Binioris, "Logistics Service Quality and Its Effects on Customer Satisfaction in the Manufacturing Companies' Supply Chains: Empirical Evidence from Greece", Journal of Modelling in Management, 9 (2014), pp. 215-237.

[37] Y.Y. Zhou, M.H. Kanter, J.J. Wang, and T. Garrido, "Improved quality at Kaiser Permanente through e-mail between physicians and patients", Health Affairs, 29 (2010), pp. 1370-1375. 